\title{
REAL ANALYTICITY OF THE CANONICAL VERSAL DEFORMATIONS OF CR-STRUCTURES
}

\author{
TAKAO AKAHORI
}

\begin{abstract}
Related to isolated singularities, Kuranishi initiated deformation theory of CR structures. And this theory has been improved in T. Akahori, 1981. In this paper, we show that the canonical family, constructed in T. Akahori, 1981, is real analytic. This result gives a direct proof of Miyajima's theorem about Isolated singularities and CR structures on their links (see K. Miyajima, 1980).
\end{abstract}

\section{Introduction.}

The purpose of this paper is to prove the real analyticity of the versal family of CR-structures, constructed in [A3]. Let $(V, o)$ be an $n$ dimensional normal isolated singularity in $\left(C^{N}, o\right)$. We set

$$
M=V \cap S_{\epsilon}^{2 N-1}(o)
$$

where $S_{\epsilon}^{2 N-1}(o)$ is the $\epsilon$ - sphere in $C^{N}$. Then we have a real odd dimensional, compact manifold, which is obviously real analytic. Furthermore, over this $M$, a CR-structure is naturally induced from $V$. By Rossi (see $[\mathbf{R}])$, this CR-structure $\left(M,{ }^{0} T^{\prime \prime}\right)$ determines the normal isolated singularity $(V, o)$, uniquely. Kuranishi noted this point, and in order to study deformation theory of isolated singularities, he initiated deformation theory of CR-structures. This method is improved by [A3], [Mi 1], [Mi 2]. Namely, in [A3], it is shown that there is a versal family in the sense of Kuranishi, $\left(M,{ }^{\phi(t)} T^{\prime \prime}\right)$ which satisfies that $\phi(t)$ is a $C^{k}$ element of $\overline{{ }^{0} T^{\prime \prime}} \otimes\left({ }^{0} T^{\prime \prime}\right)^{*}$ valued form, which depends on $t$, complex analytically, and $\phi(o)=0$. Later, Miyajima proved that $\phi(t)$ is actually of $C^{\infty}$ in [Mi 1] and discussed about the extendability of our canonical versal family in [Mi 2]. Actually, our family is versal only for deformations of tubular neighborhoods of the boundary $M$, and it was not clear that our family can be extended to the deformation of tubular neighborhoods (see [A3]). Miyajima studied an algebraic geometric approach and showed that our canonical versal family can be extended to a deformation of tubular neighborhoods of the boundary (see [Mi 2], [Mi 3]). In this paper, instead of his approach, we adopt the direct method. Namely, 
we take the compact real analytic CR manifold (for isolated singularities, this is always possible). And we show that our canonical versal family for this real analytic CR manifold, is also real analytic. Then, obviously, because of real analyticity, by using the complexfication method, our family determines a deformation of tubular neighborhoods of the boundary $M$, uniquely. Now we see our proof.

By the construction, our $\phi(t)$ satisfies the following non-linear partial differential equation.

$$
\square_{b} \phi(t)+\bar{\partial}_{b}^{*} R_{2}(\phi(t))=\square_{b} \mathcal{L}\left(\sum_{i=1}^{q} \beta_{i} t_{i}\right)
$$

$t=\left(t_{1}, . ., t_{q}\right),\left\{\beta_{i}\right\}_{1 \leq i \leq q}$ is a base of $\mathbf{H}_{T^{\prime}}^{(1)}, q=\operatorname{dim}_{C} \mathbf{H}_{T^{\prime}}^{(1)}$, (for notations and the construction, see [A3]). This nonlinear equations' principal part is subelliptic, and we note that in the nonlinear term, only $X \phi(t), X Y \phi(t)$, where $X, Y$ in ${ }^{0} T^{\prime \prime}+\overline{{ }^{0} T^{\prime \prime}}$, terms appear. Of course if there is no nonlinear term in this equation, the solution must be real analytic ( $M$ being real analytic, so real analytic hypo-ellipticity holds) (see [Tar1], [Ko]). In our case, as the non linear term is quite suitable (it doesn't include $X T \phi(t)$ term and $T T \phi(t)$ term, where $\mathrm{X}$ in ${ }^{0} T^{\prime \prime}+\overline{{ }^{0} T^{\prime \prime}}$ and $T$ is the missing direction), it is natural to expect the same result as in the elliptic case. Hence it is quite natural to follow the Tartakoff's method, which succeeded in the linear sub-elliptic case. Following the Tartakoff's method in the non linear case, we are forced to control $(X Y \phi(t)) \phi(t)$ term, where $X, Y$ in ${ }^{0} T^{\prime \prime}+\overline{{ }^{0} T^{\prime \prime}}$. However, instead of the standard $L^{2}$ norm, if we use the \|\|$_{(m)}^{\prime \prime}$ norm (see Sect. 1 in this paper), we have

$$
\|(X Y \phi(t))(\phi(t))\|_{(m)}^{\prime \prime} \leq C_{m}\|X Y \phi(t)\|_{(m)}^{\prime \prime}\|\phi(t)\|_{(m)}^{\prime \prime},(n \leq m)
$$

and moreover, our norm dosen't cause so much problem to control $\left\|T^{r} \phi(t)\right\|_{(m)}^{\prime \prime}$ and $\left\|W^{I} T^{r} \phi(t)\right\|_{(m)}^{\prime \prime}$ where $W$ in ${ }^{0} T^{\prime \prime}+\overline{{ }^{0} T^{\prime \prime}}$ (namely, the Tartakoff's method is also valid in our norm). So we have our real analyticity of our canonical family. For real analytic hypo-ellipticity, I don't prove any new particular result. However, our versal family of CR structures plays a quite important role in CR geometry. So, it is my obligation to publish our result, namely, by following the complete same method for the linear problem as in [Tar 1], even though our equation is non-linear, our versal family is real analytic.

\section{CR-structures and $E_{j}$ structures.}

We consider an n dimensional isolated singularity $(V, o)$ in $\left(C^{N}, o\right)$, and study this singularity from the point of view of CR-geometry. For this, we set a 
real analytic function on $C^{N}$,

$$
r(z)=\sum_{i=1}^{N}\left|z_{i}\right|^{2}-\epsilon
$$

where $\epsilon>0$ is chosen sufficiently small. Set

$$
M=\{x ; x \in V, r(x)=0\} .
$$

On $\mathrm{M}$, a CR structure is naturally induced from $C^{N}$. That is to say,

$$
{ }^{0} T^{\prime \prime}=\left\{X ;\left.X \in C \otimes T M \cap T^{\prime \prime} C^{N}\right|_{M}\right\} .
$$

In this paper, instead of $\overline{{ }^{0} T^{\prime \prime}}$, we use the notation ${ }^{0} T^{\prime}$. Then, our ${ }^{0} T^{\prime \prime}$ satisfies

$$
\begin{aligned}
& \text { 1) }{ }^{0} T^{\prime \prime} \cap{ }^{0} T^{\prime}=0, \operatorname{dim}_{C}\left(C \otimes T M /\left({ }^{0} T^{\prime \prime}+{ }^{0} T^{\prime}\right)\right)=1 \\
& \text { 2) }\left[\Gamma\left(M,{ }^{0} T^{\prime \prime}\right), \Gamma\left(M,{ }^{0} T^{\prime \prime}\right)\right] \subseteq \Gamma\left(M,{ }^{0} T^{\prime \prime}\right) .
\end{aligned}
$$

The pair $\left(M,{ }^{0} T^{\prime \prime}\right)$ satisfies 1$)$ and 2$)$ is called a CR structure. Now in our case, obviously, $\mathrm{M}$ is real analytic and also the induced CR structure is also real analytic. Next we set a supplement real vector field $\zeta$ by

$$
\zeta=\text { the dual vector of the real } 1 \text { form } \sqrt{-1} \partial r \text {. }
$$

So

$$
C \otimes T M={ }^{0} T^{\prime \prime}+{ }^{0} T^{\prime}+C \zeta .
$$

Next we recall $E_{j}$ structures, introduced in [A3]. For this, we set $T^{\prime}=$ ${ }^{0} T^{\prime}+C \zeta$. And set a first order differential opeator $\bar{\partial}_{T^{\prime}}$ from $\Gamma\left(M, T^{\prime}\right)$ to $\Gamma\left(M, T^{\prime} \otimes\left({ }^{0} T^{\prime \prime}\right)^{*}\right)$ by; for $\mathrm{u}$ in $\Gamma\left(M, T^{\prime}\right)$,

$$
\bar{\partial}_{T^{\prime}} u(X)=[X, u]_{T^{\prime}}
$$

for $X$ in $\Gamma\left(M,{ }^{0} T^{\prime \prime}\right)$, where $[X, u]_{T^{\prime}}$ means the $T^{\prime}$-part of $[X, u]$ according to (1.1). And like the case for scalar valued forms, we have

$$
\bar{\partial}_{T^{\prime}}^{(p)} ; \Gamma\left(M, T^{\prime} \otimes \wedge^{p}\left({ }^{0} T^{\prime \prime}\right)^{*}\right) \longrightarrow \Gamma\left(M, T^{\prime} \otimes \wedge^{(p+1)}\left({ }^{0} T^{\prime \prime}\right)^{*}\right), p=1,2, \ldots
$$

Now we set

$$
\Gamma_{p}=\operatorname{Ker} \bar{\partial}_{T^{\prime}}^{(p)} \cap \Gamma\left(M,{ }^{0} T^{\prime} \otimes \wedge^{(p)}\left({ }^{0} T^{\prime \prime}\right)^{*}\right) .
$$

Then there is a subbundle $E_{p}$ of ${ }^{0} T^{\prime} \otimes \wedge^{p}\left({ }^{0} T^{\prime \prime}\right)^{*}$ satisfying

$$
\begin{aligned}
E_{0} & =0, \\
\Gamma_{p} & =\Gamma\left(M, E_{p}\right) .
\end{aligned}
$$


And

$$
\begin{aligned}
& \bar{\partial}_{p}^{(p)} \Gamma\left(M, E_{p}\right) \subset \Gamma\left(M, E_{(p+1)}\right) \\
& \operatorname{Ker} \bar{\partial}_{b}^{(1)} \longrightarrow H^{(1)}\left(M, T^{\prime}\right) \longrightarrow 0 \\
& \frac{\operatorname{Ker} \bar{\partial}_{b}^{(p)}}{\operatorname{Im} \bar{\partial}_{b}^{(p-1)}} \simeq H^{(p)}\left(M, T^{\prime}\right), 2 \leq p \leq n-1
\end{aligned}
$$

where $\bar{\partial}_{b}^{(p)}=\left.\bar{\partial}_{T^{\prime}}^{(p)}\right|_{\Gamma\left(M, E_{i}\right)}, \operatorname{dim}_{R} M=2 n-1, \operatorname{dim}_{C}^{0} T^{\prime \prime}=n-1$. (In [A1], [A2] and [A3], we used different notations. However, in this paper, for the reader's convenience, we dare to use $\bar{\partial}_{b}^{(p)}$.) And if $n \geq 4$,

$$
\Gamma\left(M, E_{1}\right) \stackrel{\bar{\partial}_{b}}{\longrightarrow} \Gamma\left(M, E_{2}\right) \stackrel{\bar{\partial}_{b}^{(1)}}{\longrightarrow} \Gamma\left(M, E_{3}\right)
$$

is a subelliptic complex and several important estimates are proved in [A3]. (From now on, we use the notation $\bar{\partial}_{b}$ for $\bar{\partial}_{b}^{(p)}$.) We recall this. For this, we set a real 1 form $\theta$ by

$$
\begin{aligned}
\left.\theta\right|^{0} T^{\prime \prime}+{ }^{0} T^{\prime} & =0 \\
\theta(\zeta) & =1 .
\end{aligned}
$$

And we set $\omega=-\sqrt{-1} d \theta$, and then we have the Levi metric. From this metric, we define the volume element $d v$, and we set the $L^{2}$ norm on $\Gamma\left(M, E_{p}\right)$ by

$$
(u, v)=\int_{M}\langle u, v\rangle d v \quad \text { for } u, v \text { in } \Gamma\left(M, E_{p}\right)
$$

where $\langle$,$\rangle means the hermitian inner product induced from \Gamma\left(M,{ }^{0} T^{\prime} \otimes\right.$ $\left.\wedge^{p}\left({ }^{0} T^{\prime \prime}\right)^{*}\right)$. We denote $\bar{\partial}_{b}^{*}$ by the adjoint operator of $\bar{\partial}_{b}$ on $\Gamma\left(M, E_{p}\right)$ with respect to the above metric. And we set the Laplacian

$$
\square_{b}=\bar{\partial}_{b}^{*} \bar{\partial}_{b}^{*}+\bar{\partial}_{b} \bar{\partial}_{b}^{*}
$$

For $\mathrm{u}$ in $\Gamma\left(M, E_{p}\right)$, we set

$$
\begin{gathered}
\|u\|_{(m)}^{\prime \prime 2}=\sum_{i=0}^{m}\left\|\square_{b} u\right\|^{2}, \\
(u, v)_{(m)}^{\prime \prime}=\sum_{i=0}^{m}\left(\square_{b}^{i} u, \square_{b}^{i} v\right) \quad \text { for } u, v \text { in } \Gamma\left(M, E_{p}\right) .
\end{gathered}
$$

Then, we easily have: 


\section{Lemma 1.1.}

$$
\left(\bar{\partial}_{b} u, v\right)_{(m)}^{\prime \prime}=\left(u, \bar{\partial}_{b}^{*} v\right)_{(m)}^{\prime \prime} \quad \text { for } u, v \text { in } \Gamma\left(M, E_{p}\right)
$$

Namely, $\bar{\partial}_{b}^{*}$ is also the adjoint operator of $\bar{\partial}_{b}$ with respect to \|\|$_{(m)}^{\prime \prime}$. Furthermore by the result(see Proposition 3.3 in [A2]) with the standard argument, we have:

Lemma 1.2. For $u$ in $\Gamma\left(M, E_{p}\right)$,

$$
\|W W u\|_{(m)}^{\prime \prime} \leq C_{m}\|u\|_{(m+1)}^{\prime \prime} .
$$

We must explain notations. Let $\left\{U_{i}, h_{i}\right\}_{i \in I}$ be a finite set of local coordinate neighborhoods of M. And let $\{\rho\}_{i \in I}$ be a partition of unity subordinate to this covering. Let $\left\{Y_{j, k}\right\}_{1 \leq j \leq n-1}$ be an orthonormal frame of ${ }^{0} T^{\prime \prime}$ over $U_{k}$ according to the Levi metric defined by (1.1). With this preparation, the above inequality means; for u supported in $U_{k}$,

$$
\left\|W_{\alpha, k} W_{\beta, k} u\right\|_{(m)}^{\prime \prime} \leq C_{m}\|u\|_{(m+1)}^{\prime \prime},
$$

where $W_{\alpha, k}, W_{\beta, k}=Y_{j, k}$ or $\overline{Y_{j, k}}, 1 \leq j \leq n-1$. And henceforth, for this $W_{\alpha, k}$, we use the abbreviation W. Assume that $\operatorname{dim}_{R} M=2 n-1 \geq 7$. Then:

\section{Estimate (I).}

$$
\|u\|^{\prime} \leq C\left\{\left\|\bar{\partial}_{b} u\right\|+\left\|\bar{\partial}_{b}^{*} u\right\|+\|u\|\right\}, \quad \text { for } u \text { in } \Gamma\left(M, E_{2}\right)
$$

(for the notations and the proof, see Theorem 4.1 (new estimate) in [A3]). Then by the standard argument, we have the Neumann operator $N_{b}$ for the above differential complex $\left(\Gamma_{p}, \bar{\partial}_{b}^{(p)}\right)$. And so, we have the Kodaira-Hodge type decomposition theorem for this complex, namely

$$
u=H_{b} u+\square_{b} N_{b} u, \quad \text { for } u \text { in } \Gamma\left(M, E_{2}\right),
$$

where $H_{b}$ means the projection of $u$ into

$$
\left\{u ; u \text { in } \Gamma\left(M, E_{2}\right), \bar{\partial}_{b} u=0, \bar{\partial}_{b}^{*} u=0\right\} .
$$

\section{Estimate (II).}

$$
\|u\|^{\prime \prime} \leq C^{\prime}\left\{\left\|\square_{b} u\right\|+\|u\|\right\} \quad \text { for } u \text { in } \Gamma\left(M, E_{2}\right) .
$$

We note that \|\|$^{\prime \prime}$ norm is the same as \|\|$_{(0)}^{\prime \prime}$ norm introduced in this section. 


\section{Estimate (III).}

$$
\left\|W_{\alpha, k}(\rho u)\right\|_{(m)}^{\prime 2}+K\|\rho u\|_{(m)}^{\prime \prime 2} \leq C_{m}\left\{\left\|\bar{\partial}_{b}(\rho u)\right\|_{(m)}^{\prime 2}+\left\|\bar{\partial}_{b}^{*}(\rho u)\right\|_{(m)}^{\prime \prime 2}\right\}+C_{m}^{\prime}\|\rho u\|_{(m)}^{\prime \prime 2},
$$

where $W_{\alpha, k}=Y_{j, k}$ or $\bar{Y}_{j, k}$, and $\rho \in C^{\infty}$ is supported in $U_{k}$. From now on, we fix $m$, satisfying $m \geq n$.

For our norm, we have:

Lemma 1.3. Let $f, g$ be $C^{\infty}$ functions on $M$. Then,

$$
\|f g\|_{(m)}^{\prime \prime} \leq c_{m}^{\prime \prime}\|f\|_{(m)}^{\prime \prime}\|g\|_{(m)}^{\prime \prime} \text {. }
$$

The proof is standard (by using the Sobokev lemma with subelliptic estimate for $\square_{b}$ ). So, we omit this.

Finally in this section, we note that there is a real analytic real vector field $T$ on $M$ satisfying

1) $T_{p} \notin{ }^{0} T_{p}^{\prime \prime}+{ }^{0} T_{p}^{\prime} \quad$ for every point $p$ of $M$

2) $[T, Z] \equiv 0 \bmod { }^{0} T^{\prime \prime}+{ }^{0} T^{\prime} \quad$ for all $Z \in \Gamma\left(M,{ }^{0} T^{\prime \prime}+{ }^{0} T^{\prime}\right)$,

(see Proposition 1 in [Tar1]). So, using this $T$, we newly introduce a $C^{\infty}$ vector bundle decompostion

$$
C \otimes T M={ }^{0} T^{\prime \prime}+{ }^{0} T^{\prime}+C \otimes T
$$

and also introduce corresponding operators $\bar{\partial}_{T^{\prime}}, \bar{\partial}_{T^{\prime}}^{(p)}$ (as I mentioned before, we use the notation $\bar{\partial}_{b}$ for these operators). Then, the complete same results hold, and the same estimates hold. From now on, we adopt this supplement vector field $T$ and use the Levi-form according to (1.2). And following [Tar1], we use the notaion $W={ }^{0} T^{\prime \prime}+{ }^{0} T^{\prime}$.

\section{The canonical versal family.}

In this section, we recall the construction of the canonical versal family ([A3]). Namely, we set $\Gamma\left(M,{ }^{0} T^{\prime} \otimes\left({ }^{0} T^{\prime \prime}\right)^{*}\right)$ valued power series

$$
\phi(t)=\sum_{K=\left(k_{1}, . ., k_{q}\right)} \phi_{K} t_{1}^{k_{1}} \ldots . t_{q}^{k_{q}}
$$

where $t=\left(t_{1}, . ., t_{q}\right) \in U \subset C^{q}$, and $U$ is a neighborhood of the origin, and $K$ is a multi index, $q=\operatorname{dim}_{C} \mathbf{H}_{T^{\prime}}^{(1)}$. For brevity, we abbreviate this as follows.

$$
\phi(t)=\sum_{K} \phi_{K} t^{K}
$$


Now we recall the construction of $\phi(t)$. By the Banach inverse mapping theorem, we solve $\phi(t)$, namely $\phi(t)$ is the unique solution of the following.

$$
\phi(t)+\bar{\partial}_{b}^{*} N_{b} R_{2}(\phi(t))=\mathcal{L}\left(\sum_{i=1}^{q} \beta_{i} t_{i}\right), \quad\left(t_{1}, \ldots, t_{q}\right) \in U \subset C^{q},
$$

where $N_{b}$ is introduced in [A3], and $\left\{\beta_{i}\right\}_{1 \leq i \leq q}$ is a base of $\mathbf{H}_{T^{\prime}}^{(1)}$. And by [Mi 2], our $\phi(t)$ is of $C^{\infty}$. It is better to explain $\mathcal{L}$, introduced in [A2]. For $v$ in $\Gamma\left(M, T^{\prime} \otimes\left({ }^{0} T^{\prime \prime}\right)^{*}\right)$, we set

$$
\mathcal{L} v(X)=v(X)-\bar{\partial}_{b} \theta_{v}(X), \text { for } X \in \Gamma\left(M,{ }^{0} T^{\prime \prime}\right),
$$

where $T^{\prime}={ }^{0} T^{\prime}+C \otimes T$, and $\theta_{v}$ is an element of $\Gamma\left(M,{ }^{0} T^{\prime}\right)$ defined by

$$
\left[\theta_{v}, X\right]_{T}=(v(X))_{T} \text { for } X \text { in } \Gamma\left(M,{ }^{0} T^{\prime \prime}\right),
$$

where $\left[\theta_{v}, X\right]_{T}\left(\operatorname{resp} .(v(X))_{T}\right)$ means the $C \otimes T$ part of $\left[\theta_{v}, X\right](\operatorname{resp} .(\mathrm{v}(\mathrm{X})))$ according to (1.2).

\section{The real analyticity.}

As we recalled in Sect. 2, $\phi(t)$ satisfies

$$
\phi(t)+\bar{\partial}_{b}^{*} N_{b} R_{2}(\phi(t))=\mathcal{L}\left(\sum_{i=1}^{q} \beta_{i} t_{i}\right) .
$$

Hence we have

$$
\square_{b} \phi(t)+\square_{b} \bar{\partial}_{b}^{*} N_{b} R_{2}(\phi(t))=\square_{b} \mathcal{L}\left(\sum_{i=1}^{q} \beta_{i} t_{i}\right),
$$

namely

$$
\square_{b} \phi(t)+\bar{\partial}_{b}^{*} R_{2}(\phi(t))=\square_{b} \mathcal{L}\left(\sum_{i=1}^{q} \beta_{i} t_{i}\right) .
$$

First, by the real analyticity of the solution to $\square_{b}, \beta_{i}$ must be real analytic. We show that this $\phi(t)$ is real analytic. We follow the Tartakoff's line in [Tar1] and we adopt his notations. Let $p_{o}$ be the reference point of $M$. Let $U_{1}\left(p_{o}\right)$ be a sufficiently small neighborhood of $p_{o}$ in $\mathrm{M}$, and $U_{2}\left(p_{o}\right)$ be a neighborhood of $p_{o}$ satisfying; $U_{1}\left(p_{o}\right) \Subset U_{2}\left(p_{o}\right)$. And we fix an integer $m$ satisfying; $m \geq n$. 
Now we show that there are constants $C_{1}$ and $C_{2}$ which satisfy; there is a $\epsilon>0$, and for every $q$, there is a $C^{\infty}$ function $\psi_{q}$ supported in $U_{2}\left(p_{o}\right)$ and $\left.\psi_{q}\right|_{U_{1}\left(p_{o}\right)}=1$ satisfying

$$
\left\|\psi_{q} O p(q) \phi(t)\right\|_{(m)}^{\prime \prime} \leq C_{1} C_{2}^{q} q !, \quad \text { for any } t \text { in }(0, \epsilon) .
$$

Here $O p(q)$ denotes the q-th order differential operator formed by $T, W_{j}$ in $W$. If this is proved, by the Sobolev lemma, for every q,

$$
\operatorname{Sup}_{U_{1}\left(p_{o}\right)}|O p(q) \phi(t)| \leq c\|O p(q) \phi(t)\|_{(m), U_{1}\left(p_{o}\right)}^{\prime \prime}, \quad(\text { by } m \geq n),
$$

where \|\|$_{(m), U_{1}\left(p_{o}\right)}^{\prime \prime}$ means the corresponding norm over $U_{1}\left(p_{o}\right)$. So

$$
\begin{aligned}
\operatorname{Sup}_{U_{1}\left(p_{o}\right)}|O p(q) \phi(t)| & \leq c\left\|\psi_{q} O p(q) \phi(t)\right\|_{(m)}^{\prime \prime} \\
& \leq c C_{1} C_{2}^{q} q !
\end{aligned}
$$

Therefore by Lemma 1 in [Tar1], we have that $\phi(t)$ is real analytic for any $t$ in $(0, \epsilon)$. And by the following lemma, it is shown that $\phi(t)$ is real analytic.

Lemma 3.1. Let $u(x, t)$ is a $C^{k}$ function on $R^{l} \times C^{n}(k \geq 1)$, which is real analytic with respect to $x$, and complex analytic with respect to $t$, separately. Then, $u(x, t)$ is real analytic on $(x, t)$.

Proof. We consider the partial complexfication of $R^{l} \times C^{n}, C^{l} \times C^{n}$. And for a fixed $t$, we can naturally consider $\widetilde{u(z, t)}$ on $C^{l} \times C^{n}$ for $u(x, t)$. By the assumption, our $\widetilde{u(z, t)}$ is complex analytically with respect to respectively $z$ and $t$. So by Osgood's lemma, our $\widetilde{u(z, t)}$ is complex analutic with respect to both variables. So $u(x, t)$ must be real analytic.

For $(*)$, it suffices to show; there are constants $C_{1}$ and $C_{2}$ which satisfy; there is a $\epsilon>0$, and for every $q$, there is a $C^{\infty}$ function $\psi_{q}$ supported in $U_{2}\left(p_{o}\right)$ and $\left.\psi_{q}\right|_{U_{1}\left(p_{o}\right)}=1$ satisfying

$$
\left\|\psi_{q} W^{I} T^{r} \phi(t)\right\|_{(m)}^{\prime \prime} \leq C_{1} C_{2}^{|I|+r}|I| ! r !, \quad \text { for } \quad|t|<\epsilon
$$

(see Proposition 1 in [Tar2]). We see the proof of $(* *)$. In order to see this, we recall several lemmas which were shown in [Tar1], and use his useful notations. Following [Tar1], $O p(k, q)$ denotes a $q$-th order differential operator formed by concatenating $k W$ 's and $q-k T^{\prime}$ s.

Lemma 3.2 (Lemma 2 in [Tar1]). For $k \geq 1$, any $O p(k, q)$ may be written sybolically

$$
O p(k, q)=W O p(k-1, q-1)+\sum_{j=1}^{q} c^{j}\left(\begin{array}{l}
q \\
j
\end{array}\right) a_{(j)} O o(k, q-j), \quad \text { i.e. }
$$


if there is a $W$, we may commute it to the left modulo the indicated sum of at most $c^{j}\left(\begin{array}{l}q \\ j\end{array}\right)$ terms, c some integer depending only on $n$, of the form $a_{(j)} O p(k, q-j)$.

Lemma 3.3 (Lemma 3 in [Tar1]). Let a denote any of a finite number of real analytic functions and $Z$ any of a finite number of real analytic vector field. Let $\left\{a_{(q)}\right\}$ be recursively defined by

$$
\begin{aligned}
a_{(1)} & =\text { any of the } a^{\prime} s \\
a_{(q+1)} & =a_{(1)} a_{(q)} \text { or } Z a_{(q)},
\end{aligned}
$$

i.e., $a_{(1)} a_{(q)}$ stands for one of the a's times an expression of the form $a_{(q)}$. Then locally there exists $K$ such that for all $\alpha$ and for all $q$,

$$
\left|D^{\alpha} a_{(q)}\right| \leq K K^{(|\alpha|+q)}(|\alpha|+q) !
$$

Then, as for our norm, we immediately have:

\section{Lemma 3.4.}

$$
\left\|D^{\alpha} a_{(q)}\right\|_{(m)}^{\prime \prime} \leq K^{\prime} K^{\prime(|\alpha|+q+2 m)}(|\alpha|+q+2 m) ! .
$$

So by choosing a proper $K$, we have

$$
\left\|D^{\alpha} a_{(q)}\right\|_{(m)}^{\prime \prime} \leq K K^{(|\alpha|+q)}(|\alpha|+q) !
$$

Lemma 3.5 (Lemma 4 in $[\operatorname{Tar} 1])$.

$$
\left[T^{r}, \square_{b}\right]=\sum_{j=1}^{r} c^{j}\left(\begin{array}{l}
r \\
j
\end{array}\right)\left\{W a_{(j+1)} W+W a_{(j+2)}+a_{(j+3)}\right\} T^{r-j} .
$$

Now we begin by estimating $\left\|\rho W T^{p} \phi(t)\right\|_{(m)}^{\prime \prime}$ and $\left\|\rho T^{p} \phi(t)\right\|_{(m)}^{\prime \prime}$. Namely we show the following proposition. By a technical reason, we change the estimate. The following estimate is not the same as in $(* *)$. But by choosing $C_{1}, C_{2}$ properly, it is equivalent to $(* *)$ in the case $|I| \leq 1$.

Proposition 3.6. If we choose $C_{1}, C_{2}$ sufficiently large,

$$
\left\|\rho W T^{p} \phi(t)\right\|_{(m)}^{\prime \prime} \text { and }\left\|\rho T^{p} \phi(t)\right\|_{(m)}^{\prime \prime} \leq C_{1} C_{2}^{p-2}(p-2) ! \text { for every } p \geq 2 \text {. }
$$

Proof. Our problem is on a compact manifold without boundary. So, by following Tartakoff's method in [Tar1], we can omit $\rho$. We show this estimate 
by induction on $p$. The case $p=2$ is no problem if we choose $C_{1}$ sufficiently large. We assume $p=k$ case. And show $p=k+1$ case. We recall the basic estimate (Estimate (III) without $\rho$ ).

$$
\|W u\|_{(m)}^{\prime \prime 2}+K\|u\|_{(m)}^{\prime \prime 2} \leq c_{m}\left\{\left\|\bar{\partial}_{b} u\right\|_{(m)}^{\prime \prime 2}+\left\|\bar{\partial}_{b}^{*} u\right\|_{(m)}^{\prime 2}\right\}+C_{K, m}\|u\|^{2}
$$

namely,

$$
\leq c_{m}\left\{\sum_{i=0}^{m}\left(\square_{b}^{i} \bar{\partial}_{b} u, \square_{b}^{i} \bar{\partial}_{b} u\right)+\sum_{i=0}^{m}\left(\square_{b}^{i} \bar{\partial}_{b}^{*} u, \square_{b}^{i} \bar{\partial}_{b}^{*} u\right)\right\}+C_{K, m}\|u\|^{2} .
$$

So in the place of $u$ in this equality, we put $u=T^{k+1} \phi(t)$. Then, we have

$$
\begin{aligned}
& \left\|W\left(T^{k+1} \phi(t)\right)\right\|_{(m)}^{\prime \prime 2}+K\left\|T^{k+1} \phi(t)\right\|_{(m)}^{\prime 2} \\
& \leq c_{m}\left\{\sum_{i=0}^{m}\left(\square_{b}^{i} \bar{\partial}_{b}\left(T^{k+1} \phi(t)\right), \square_{b}^{i} \bar{\partial}_{b}\left(T^{k+1} \phi(t)\right)\right)\right. \\
& \left.+\sum_{i=0}^{m}\left(\square_{b}^{i} \bar{\partial}_{b}^{*}\left(T^{k+1} \phi(t)\right), \square_{b}^{i} \bar{\partial}_{b}^{*}\left(T^{k+1} \phi(t)\right)\right)\right\}+C_{K, m}\left\|T^{k+1} \phi(t)\right\|^{2}
\end{aligned}
$$

$(* * *)$

$$
\leq c_{m}\left\{\sum_{i=0}^{m}\left(\square_{b}^{i} \square_{b}\left(T^{k+1} \phi(t)\right), \square_{b}^{i}\left(T^{k+1} \phi(t)\right)\right)\right\}+C_{K, m}\left\|T^{k+1} \phi(t)\right\|^{2} .
$$

Furthermore

$$
\begin{aligned}
\left(\square_{b}^{m} \square_{b} T^{k+1} \phi(t), \square_{b}^{m} \square_{b} T^{k+1} \phi(t)\right) \\
=\left(\square_{b}^{m} T^{k+1} \square_{b} \phi(t), \square_{b}^{m} T^{k+1} \square_{b} \phi(t)\right)+\left(\square_{b}^{m}\left[\square_{b}, T^{k+1}\right] \phi(t), \square_{b}^{m} T^{k+1} \phi(t)\right) \\
=\left(\square_{b}^{m} T^{k+1} \square_{b} \phi(t), \square_{b}^{m} T^{k+1} \phi(t)\right) \\
\quad+\left(\square_{b}^{m} W\left(\sum_{j=1}^{k+1} a_{(j+1)} W T^{k+1-j} \phi(t)\right), \square_{b}^{m} T^{k+1} \phi(t)\right), \text { (by Lemma 3.5). }
\end{aligned}
$$

By the same way as in [Tar1], we can handle the second term of the above. So we omit this. We will control the first term. We operate $T^{k+1}$ on (3.0.1). Then, we have

$$
T^{k+1} \square_{b} \phi(t)+T^{k+1} \bar{\partial}_{b}^{*} R_{2}(\phi(t))=T^{k+1} \square_{b} \mathcal{L}\left(\sum_{i=1}^{q} \beta_{i} t_{i}\right) .
$$

And so by this equation, the first term of the above can be estimated by;

$$
\begin{aligned}
& \left|\left(\square_{b}^{m} T^{k+1} \square_{b} \mathcal{L}\left(\sum_{i=1}^{q} \beta_{i} t_{i}\right), \square_{b}^{m} T^{k+1} \phi(t)\right)\right| \\
& +\left|\left(\square_{b}^{m} T^{k+1} \bar{\partial}_{b}^{*} R_{2}(\phi(t)), \square_{b}^{m} T^{k+1} \phi(t)\right)\right| .
\end{aligned}
$$


The first term of this was already handled by [Tar1]. We see the second term which didn't appear in [Tar1]. The second term becomes

$$
\begin{aligned}
& \left|\left(\square_{b}^{m} T^{k+1} R_{2}(\phi(t)), \square_{b}^{m} \bar{\partial}_{b} T^{k+1} \phi(t)\right)\right|+\text { commutator terms } \\
& \leq \text { (large constant) }\left\|\square_{b}^{m} T^{k+1} R_{2}(\phi(t))\right\|^{2} \\
& \quad+\text { (small constant) }\left\|\square_{b}^{m} \bar{\partial}_{b} T^{k+1} \phi(t)\right\|^{2} \\
& \quad+\text { commutator terms. }
\end{aligned}
$$

To control commutator terms is not easy. But the method is standard. So we omit this. For (small constant) $\left\|\square_{b}^{m} \bar{\partial}_{b} T^{k+1} \phi(t)\right\|^{2}$,

$$
\text { (small constant) }\left\|\square_{b}^{m} \bar{\partial}_{b} T^{k+1} \phi(t)\right\|^{2}=\text { (small constant) }\left\|\bar{\partial}_{b} T^{k+1} \phi(t)\right\|_{(m)}^{\prime 2} .
$$

So this term can be absorbed in $\left\|W\left(T^{k+1} \phi(t)\right)\right\|_{(m)}^{\prime 2}$, namely, the left hand side of $(* * *)$. Therefore we can ignore this term for our estimate if we choose $C_{1}, C_{2}$ properly. For the non-linear term $T^{k+1} R_{2}(\phi(t))$ :

Lemma 3.7. If we choose $C_{1}, C_{2}$ sufficiently large, we have

$$
\begin{aligned}
\left\|T^{k+1} R_{2}(\phi(t))\right\|_{(m)}^{\prime \prime} \leq & \left(\frac{1}{4}\right) C_{1} C_{2}^{k-1}(k-1) ! \\
& +(\text { small constant })\left\|W\left(T^{k+1} \phi(t)\right)\right\|_{(m)}^{\prime 2} \\
& +(\text { small constant })\left\|\left(T^{k+1} \phi(t)\right)\right\|_{(m)}^{\prime 2}
\end{aligned}
$$

where (small constant) doesn't depend on $C_{1}, C_{2}$.

Proof. To estimate $T^{k+1} R_{2}(\phi(t))$, it suffices to estimate $T^{k+1}\{(W \phi(t)) \phi(t)\}$ because of the definition of $R_{2}(\phi(t))$ (see [A3]). Namely,

$$
\begin{aligned}
T^{k+1}\{(W \phi(t)) \phi(t)\}= & \left(T^{k+1}(W \phi(t))\right) \phi(t)+\left(\begin{array}{c}
k+1 \\
1
\end{array}\right)\left(T^{k}(W \phi(t))\right)(T \phi(t)) \\
& +\left(\begin{array}{c}
k+1 \\
k
\end{array}\right)(T(W \phi(t)))\left(T^{k} \phi(t)\right)+(W \phi(t))\left(T^{k+1} \phi(t)\right) \\
& \left.+\sum_{r=2}^{k-1}\left(\begin{array}{c}
k+1 \\
r
\end{array}\right)\left(T^{r}(W \phi(t))\right)\left(T^{k+1-r} \phi(t)\right)\right) .
\end{aligned}
$$

Since $\phi(o)=0$, we can assume that $\|\phi(t)\|_{(m)}^{\prime \prime},\|W \phi(t)\|_{(m)}^{\prime \prime}$ and $\|T \phi(t)\|_{(m)}^{\prime \prime}$ are sufficiently small if we choose $\epsilon$ sufficiently small (we are considering on $|t|<\epsilon)$ ( $m$ is fixed). So the first term and the fourth term can be absorbed in 
(small constant) $\left\|W\left(T^{k+1} \phi(t)\right)\right\|_{(m)}^{\prime \prime 2}+\left(\right.$ small constant) $\left\|T^{k+1} \phi(t)\right\|_{(m)}^{\prime 2}$. The second term and the third term are also no problem. In fact, if $\|\phi(t)\|_{(m)}^{\prime \prime}$, $\|W \phi(t)\|_{(m)}^{\prime \prime}$ and $\|T \phi(t)\|_{(m)}^{\prime \prime}$ are sufficiently small and we choose $C_{1}, C_{2}$ properly with the assumption $p=k$ case, these don't bother us. We see how to control the other term. By induction, we see

$$
\left\|T^{l}(W \phi(t))\right\|_{(m)}^{\prime \prime},\left\|T^{l} \phi(t)\right\|_{(m)}^{\prime \prime} \leq C_{1} C_{2}^{l-2}(l-2) !, \quad \text { if } k+1 \geq l \geq 2 .
$$

$l=2$ case is $\mathrm{OK}$, if we choose $C_{1}$ sufficiently large. We assume $l=p-1$ case. Now we see $p$ case. By $m \geq n$

$$
\begin{aligned}
& \left\|\sum_{r=2}^{p-2}\left(\begin{array}{l}
p \\
r
\end{array}\right)\left(T^{r}(W \phi(t))\right)\left(T^{p-r} \phi(t)\right)\right\|_{(m)}^{\prime \prime} \\
& \leq \sum_{r=2}^{p-2}\left(\begin{array}{l}
p \\
r
\end{array}\right) c_{m}^{\prime \prime}\left\|T^{r}(W \phi(t))\right\|_{(m)}^{\prime \prime}\left\|T^{p-r} \phi(t)\right\|_{(m)}^{\prime \prime} \\
& \leq \sum_{r=2}^{p-2}\left(\begin{array}{l}
p \\
r
\end{array}\right) c_{m}^{\prime \prime} C_{1} C_{2}^{r-2}(r-2) ! C_{1} C_{2}^{p-r-2}(p-r-2) ! \\
& \leq \sum_{r=2}^{p-2}\left(\begin{array}{l}
p \\
r
\end{array}\right) c_{m}^{\prime \prime} C_{1}^{2} C_{2}^{p-4}(r-2) !(p-r-2) !
\end{aligned}
$$

And

$$
\sum_{r=2}^{p-2}\left(\begin{array}{l}
p \\
r
\end{array}\right)(r-2) !(p-r-2) !=\sum_{r=2}^{p-2} \frac{p(p-1)(p-2) !}{r(r-1)(p-r)(p-r-1)} .
$$

While if $r \leq\left[\frac{p}{2}\right], p-r \geq \frac{p}{2}, p-r-1 \geq \frac{p}{2}$. Hence

$$
\begin{aligned}
& \sum_{r=2}^{p-2} \frac{p(p-1)(p-2) !}{r(r-1)(p-r)(p-r-1)} \\
& =\sum_{r=2}^{\left[\frac{p}{2}\right]} \frac{2 p(p-1)(p-2) !}{r(r-1)(p-r)(p-r-1)} \\
& \leq \sum_{r=2}^{\left[\frac{p}{2}\right]} \frac{8}{r(r-1) p^{2}} \times p(p-1)(p-2) ! \\
& \leq 8 \frac{p-1}{p}(p-2) ! \\
& \leq 8(p-2) !
\end{aligned}
$$

Hence

$$
(3.7 .1) \leq\left(\frac{8 C_{1} c_{m}^{\prime \prime}}{C^{2}}\right) C_{1} C_{2}^{p-2}(p-2) !
$$


So if we choose $\frac{8 C_{1} c_{m}^{\prime \prime}}{C_{2}^{2}} \leq 1$, then we have our estimate. Therefore by summing up these, we obtain the corresponding estimate for

$$
\left\|T^{k+1} \phi(t)\right\|_{(m)}^{\prime \prime},\left\|W T^{k+1} \phi(t)\right\|_{(m)}^{\prime \prime} \text {. }
$$

For $\left\|W^{I} T^{p} \phi(t)\right\|_{(m)}^{\prime \prime},|I| \geq 2$, following the Tartakoff's method, namely using Ehrenpreis's localizing function with careful study of the non-linear term in the same way as in Lemma 3.7 (we have to estimate $\left\|W^{I} T^{p}\left(R_{2}(\phi(t))\right)\right\|_{(m)}^{\prime \prime 2}$, however this is proved in the same way), we have the real analyticity of $\phi(t)$.

\section{References}

[A1] T. Akahori, Intrinsic formula for Kuranishi's $\bar{\partial}_{b}^{\phi}$, Publ. RIMS, 14 (1978), 615-641.

[A2] Complex analytic construction of the Kuranishi family on a normal strongly pseudo convex manifold, Publ. RIMS, 14 (1978), 789-847.

[A3] - The new estimate for the subbundles $E_{j}$ and its application to the deformation of the boundaries of strongly pseudo convex domains, Invent. math., 63 (1981), 311-334.

[Ko] G. Komatsu, Global analytic hypoellipticity of the $\bar{\partial}-$ Neumann problem, Tohoku Math. J. Ser.2, 28 (1976), 145-156.

$[\mathrm{Ku}] \mathrm{M}$. Kuranishi, Deformations of isolated singularities and $\bar{\partial}_{b}$, preprint.

[Mi 1] K. Miyajima, Completion of Akahori's construction of the versal family of strongly pseudo convex CR structures, Trans. Amer. Math. Soc., 277 (1980), 162-172.

[Mi 2] D Deformations of a complex manifold near a strongly pseudo-convex real hypersurface and a realization of Kuranishi family of strongly pseudo-convex $C R$ structures, Math. Z., 205 (1990), 593-602.

[Mi 3] - On realizations of families of strongly pseudo-convex CR structures, Tokyo J. Math., 15(1) (1992), 153-170.

[R] H. Rossi, Isolated singularities, Proceedings of KATATA conference, (1966), 96-102.

[Tar1] D. Tartakoff, On the global real analyticity of the solution to $\square_{b}$ on compact manifolds, Comm. in Part. Diff. Eqs., 4 (1976), 283-311.

[Tar2] - The local real analytic solutions to $\square_{b}$ and the $\bar{\partial}$-Neumann problem, Acta. math., 145 (1980), 117-204.

Received March 4, 1996.

Himeji Institute of Technology

SHOSYA 2167

HIMEJI 671-22, JAPAN

E-mail address: akahorit@sci.himeji-tech.ac.jp 